\title{
Identification of air and sea-surface targets with a Laser Range Profiler
}

\author{
Johan C. van den Heuvel, Robin M. Schoemaker, and Ric (H.)M.A. Schleijpen \\ TNO Defence, Security and Safety, Oude Waalsdorperweg 63, The Hague, The Netherlands
}

\begin{abstract}
Current coastal operations have to deal with threats at short range in complex environments with both neutral and hostile targets. There is a need for fast identification, which is possible with a laser range profiler. A number of field trials have been conducted to validate the concept of identification with a laser range profile. A laser range profiler with a high bandwidth, fast laser receiver was used to perform tests on the capability of a laser range profiler for ship identification. Typical rise and fall times are $2 \mathrm{~ns}$ corresponding to a range resolution of 0.6 meter. The experimental profiles of the ships and simulated range profiles based on 3D target models show very good correspondence. It is shown that laser range profiles match closely the geometric structure of the ship. Furthermore, the good match between experimental and simulated laser range profiles means that a database of laser range signatures can be constructed from 3D-models, thus simplifying the database creation. Based on the experiments a system model was made for the range profiling of air targets. The validated system model shows that air targets can be identified at ranges of several tens of kilometers. An identification algorithm was used to distinguish three aircraft from their simulated range profile with good results.
\end{abstract}

Keywords: Laser, littoral, range profile, identification, classification

\section{INTRODUCTION}

Maritime borders and coastal zones are susceptible to threats like drug trafficking, piracy, undermining economical activities, etc. Effective surveillance of maritime borders and coastal zones is a necessity when they are subject to such threats. A laser range profiler can provide identification capability in combination with a detection sensor like radar. The detection sensor gives the location of the threat, while the laser range profiler can identify the target based on high range-resolution data.

A similar application of a laser range profiler is in combination with an Infrared Search and Track (IRST) sensor on board of a naval vessel. Here the profiler adds identification capability of sea and air targets to the detection capability of an IRST. In addition, the profiler gives the range and can discriminate between false alarms and potential threats. False alarms that can limit IRST usefulness like birds, cloud edges, and sun glints can be easily discriminated from real targets by a laser range profiler.

A laser range profiler (LRP) has the favorable properties of a good range resolution in combination with low sea-surface clutter. These properties have been validated by experiments with an eye-safe $1.5 \mu \mathrm{m}$ laser range profiler. A range resolution of $0.6 \mathrm{~m}$ has been achieved. Sea-surface reflection was negligible. ${ }^{7}$ Identification with laser range profiles is in principle easier than for radar range profiles since radar returns depend very sensitive on aspect angle due to the predominant specular reflection. ${ }^{1,2}$

In addition, laser range profiling is an attractive option for air-target identification in an air-to-air scenario. A range profile of the aircraft is obtained from the reflection of the short laser pulse by the aircraft. This range profile is the basis for identification. Simulation shows that air targets can be distinguished from their range profiles. Identification has been tested on simulated range profiles of a number of air targets. With a range resolution of 0.3 meter, comparable to existing demonstrator systems, these air targets could be identified.

Laser Radar Technology and Applications XIV, edited by Monte D. Turner, Gary W. Kamerman, Proc. of SPIE Vol. 7323, 73230Y · ( ) 2009 SPIE · CCC code: 0277-786X/09/\$18 · doi: 10.1117/12.818426 


\section{EXPERIMENTAL SET-UP}

An experimental laser range profiler was used to validate the usefulness of such a system for sea-surface target identification. The system is depicted in Figure 1. The green box contains the OPO laser wavelength converter, which converts the pump laser wavelength of $1.064 \mu \mathrm{m}$ into the more eye-safe wavelength of $1.57 \mu \mathrm{m}$. The white box on top of the green box contains the detector and receiver telescope. The white box right next to the green box is the $1.064 \mu \mathrm{m}$ pump laser.

The pump laser for the OPO is a Q-switched Nd:YAG laser. The maximum output is $350 \mathrm{~mJ}$ per pulse with a pulse length of $5 \mathrm{~ns}$ at a rep-rate of $20 \mathrm{~Hz}$. In the OPO the laser radiation at $1.064 \mu \mathrm{m}$ is converted to $1.57 \mu \mathrm{m}$. The conversion takes place in a non-linear crystal. To increase the non-linear conversion efficiency it is beneficial to increase the laser power density by focusing the laser beam into the crystal. However there is a limit on the maximum power density that the crystal can withstand.

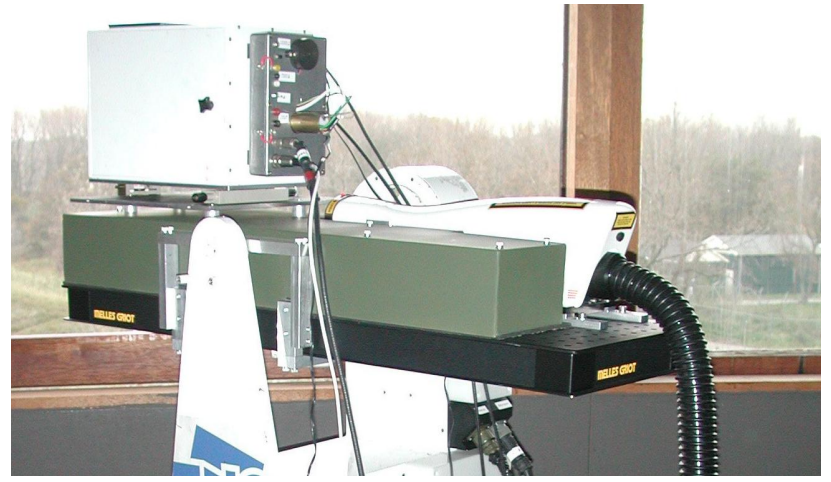

Figure 1: 1.5 micron lidar system

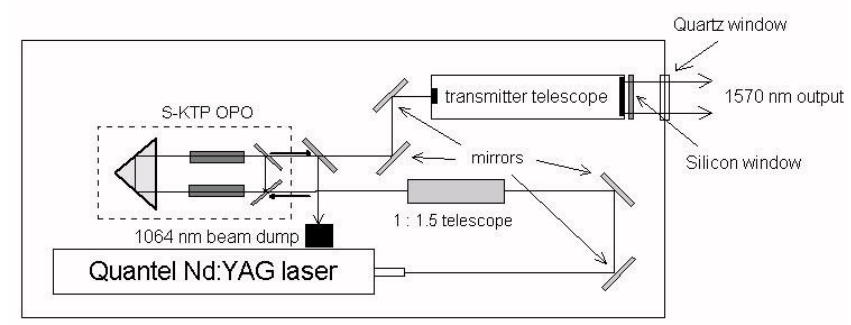

Figure 2: Optical layout of eye safe lidar transmitter.

We have made an OPO design that maximizes the non-linear conversion efficiency by using two crystals instead of one, and by using S-KTP instead of KTP crystals, which have a higher damage threshold. We ended up with a ring cavity design. The OPO laser is connected to an optical telescope and in addition some filters are placed in the beam line to completely block the eye-hazardous laser pump light. The layout of the transmit section of the lidar is shown in Figure 2. The lidar system was operated with maximum pump pulse energy of $205 \mathrm{~mJ}$ at a rep-rate of $10 \mathrm{~Hz}$. The output from the transmitter at $1570 \mathrm{~nm}$ was around $30 \mathrm{~mJ}$.

The receiver is constructed using a $750 \mathrm{~mm}$ telescope, with an optical aperture of $125 \mathrm{~mm}$ diameter. An InGaAs APD detector with a diameter of $80 \mu \mathrm{m}$ was used. The small diameter APD detector is used because it allows a very high electrical bandwidth $(200 \mathrm{MHz})$, which is required for a high range-resolution (0.6 meter).

\section{EXPERIMENTAL RESULTS}

Laser Range (LR) profiles of the various ships could be obtained at long range up to $10 \mathrm{~km}$ during the NATO MCG/8 trial in Norway, 2007. Based on these data, we will now investigate whether the experimental LR profiles correspond with simulated LR profiles based on a geometrical 3D model of a frigate. This is important in view of the results of ref. [8] that were based solely on simulated data. In that paper identification of ships with a LRP was presented based on simulated data. In addition, simulated LR profiles could be a convenient way to obtain a database of LR profiles of surface targets. Since in most cases a geometric model of a ship exists or can be constructed from photographs, a database of LR profiles based on such a model can be constructed.

A simulated range profile was obtained from an in-house electro-optics model (EOSTAR ${ }^{6}$ ) and the 3D model. In this example the ship was illuminated from the front side. Diffuse Lambertian reflection was assumed for all surfaces. ${ }^{3,4,5}$ The effect of diffuse reflection was calculated in Matlab. Figure 3 shows the 3D points used by Matlab to calculate the 
range profile. Note that the longest axis corresponds to the range. The reflection from the sea surface was assumed negligible, which has been confirmed by several experiments in the past near the coast. ${ }^{7}$ Furthermore, the MCG/8 trial shows negligible surface reflection as well.

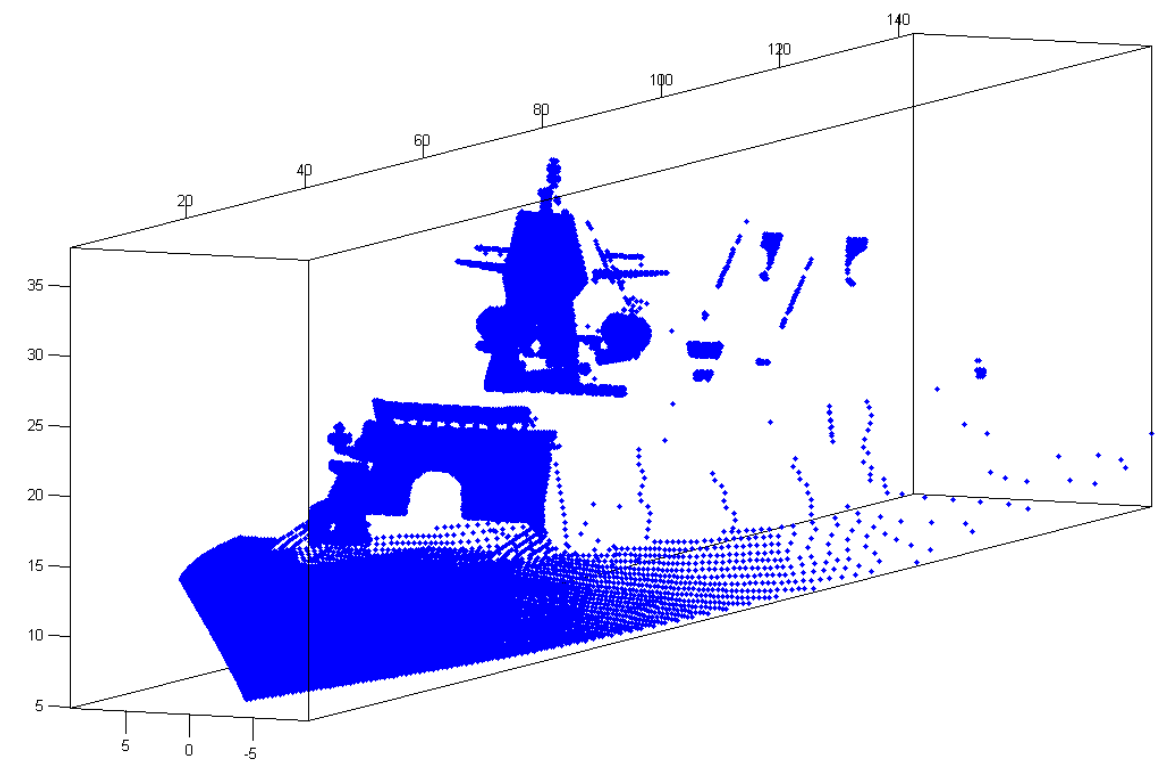

Figure 3: Plot of data points used in Matlab to generate LR profile.

Using the Matlab simulation model, the simulated LR profile of a frigate with front side laser illumination is obtained. Figure 4 shows the simulated LR profile. In addition, the experimental LR profile and a plot with both profiles are shown. It is clear that the simulated LR profile corresponds quite accurately to the experimental one. Note that not only the position of the peaks corresponds but also the height of the peaks. However, the combined plot shows a difference between the experimental and the simulated LR profiles, which is indicated by an oval in the figure.

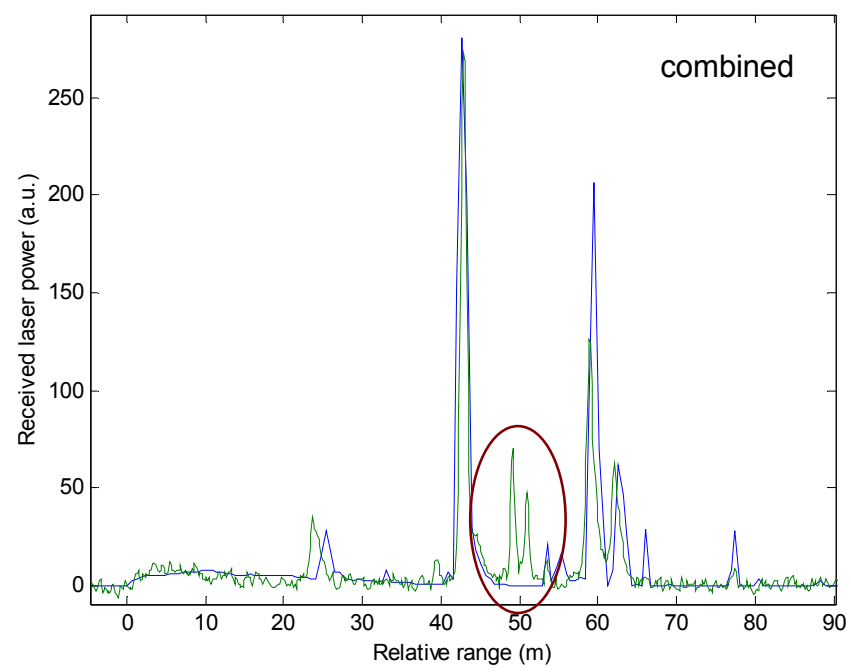

Figure 4: Comparison of experimental and simulated LR profiles of a frigate. The experimental profile is indicated by a green line and the simulated by a blue line. The oval indicates a difference between experiment and simulation.

The difference between the experimental and the simulated range profile can be understood by comparing these profiles with a photograph taken at short range of the ship at the MCG/8 trial. The photographs in Figure 5 clearly show the 
structures that show up in the experimental profile but are absent in the simulated profile. In the 3D model these structures above the bridge are absent. Thus, the difference between experiment and simulation is caused by an incomplete 3D model.

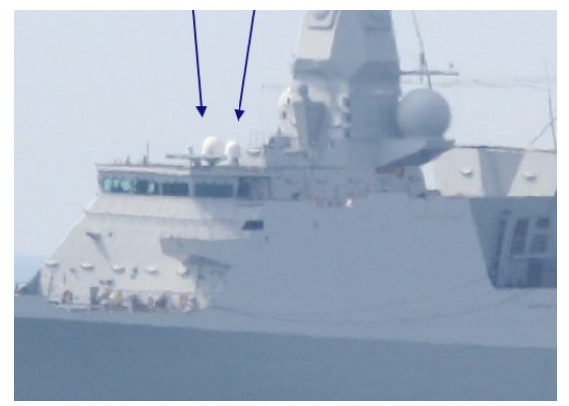

Figure 5: Satcom structures responsible for peaks in the experimental LR profile that are not present in the simulated LR profile.

\section{SIMULATED PROFILES OF AIR TARGETS}

For the study of air-target identification with a laser range profiler, we use simulated profiles obtained using 3D computer models. We have used 3D models for three aircraft: an F15, an F16 and an F117. In Figure 6 we show pictures of the aircraft that have been made on basis of the facet models and in Table 1 we summarize the main dimensions of the aircraft.
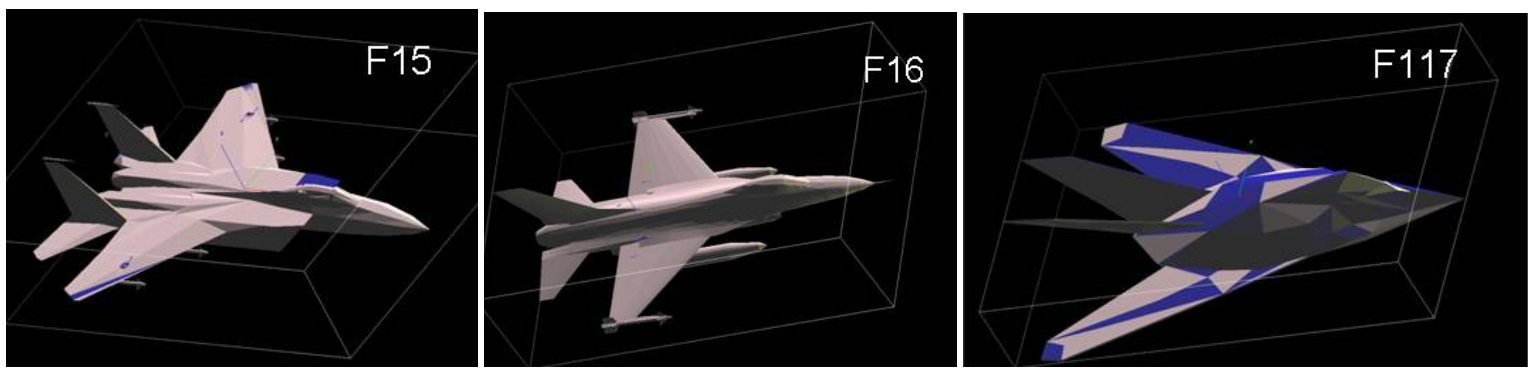

Figure 6: Pictures of the three aircraft

Table 1 The dimensions in meters of these targets.

\begin{tabular}{|l|l|l|l|}
\hline & length & wingspan & height \\
\hline F15 & 19.4 & 13.1 & 5.6 \\
\hline F16 & 14.8 & 9.8 & 4.8 \\
\hline F117 & 20.1 & 13.2 & 3.8 \\
\hline
\end{tabular}

For the laser range profiler, simulated range profiles are obtained from an in-house electro-optics model (EOSTAR $\left.{ }^{6}\right)$ and the target facet models. Diffuse Lambertian reflection was assumed for all surfaces. ${ }^{3-5}$ The effect of diffuse reflection and the receiver noise was calculated in Matlab.

Figure 7 shows the range profiles as a function of target aspect angle. A single range profile is shown by an intensity plot along a single horizontal line. Every horizontal line represents a different aspect angle. The small aircraft pictures on the right illustrate five different orientations: 0, 45, 90, 135, and 180 degrees. As will be shown later, a database of range profiles at various orientations is used for identification. 

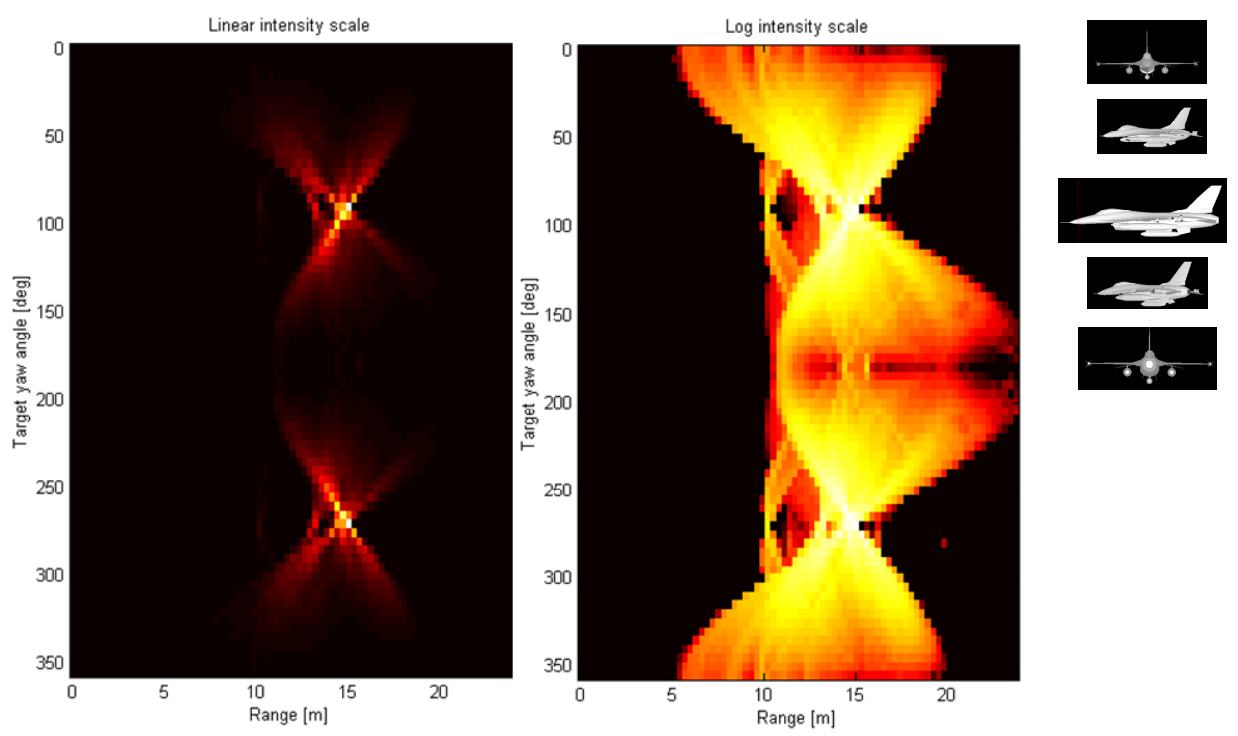

Figure 7: Simulated LR profiles of an F16 for various target aspect angles (left linear intensity scale, right logarithmic scale).

\section{AIR-TO-AIR IDENTIFICATION SCENARIO}

The following scenario is defined. Two aircraft are approaching each other with a speed of about 0.9 Mach at high altitude. A laser range profiling sensor is mounted on the observation aircraft. The two aircraft are approaching at an angle of about 90 degrees. We consider the atmospheric conditions as optimal. Detection and tracking of the aircraft is based on radar or IRST. Laser range profiling is not used for detection, but only for identification. Thus the precise location of the aircraft is known and a laser can be aimed at the air target.

For the identification study, laser range profiles are simulated based on the target range and aspect angle in the scenario. The simulation of the laser range profiles is based on currently available technology with a range resolution of $30 \mathrm{~cm}$. From the scenario, observation parameters like distances and angles (elevation and azimuth) are extracted. Using the 3D models, simulated sensor data are obtained for the laser range profiler observing the three targets. Figure 9 shows the simulated laser range profiles for an approaching F16 according to the scenario.

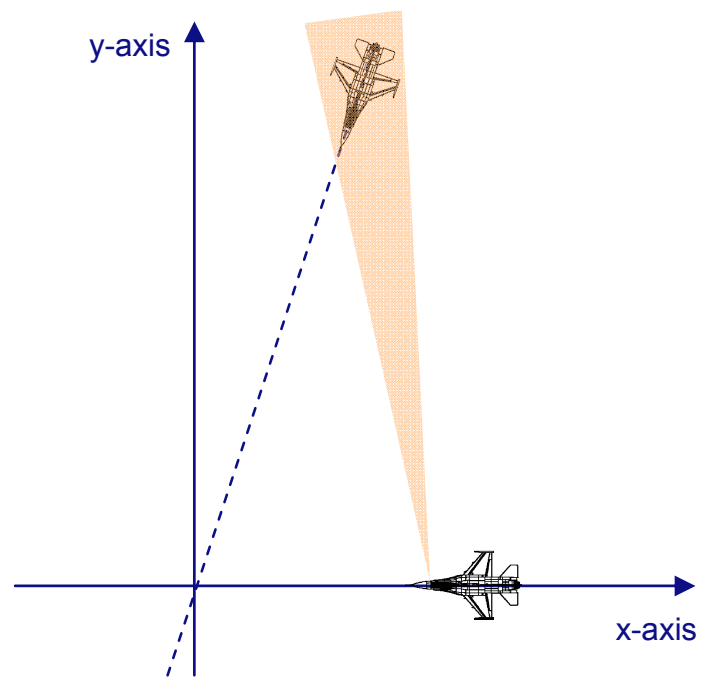

Figure 8: Layout of scenario with two approaching aircraft. 


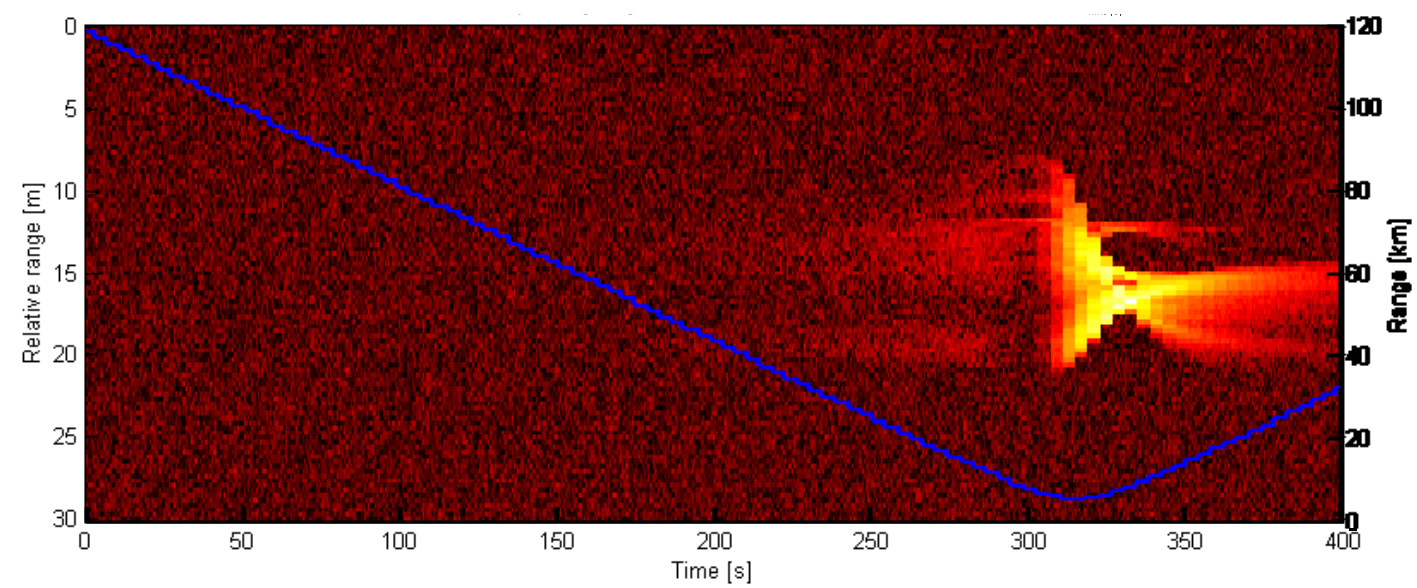

Figure 9: Simulated laser range profiles for an approaching F16 according to the scenario. Every image column represents a single profile on a linear intensity scale. The blue line indicates the distance corresponding to the right axis.

These laser range profiles have $30 \mathrm{~cm}$ range resolution. Noise has been added so that the signal at distances above $10 \mathrm{~km}$ is dominated by noise. A later section on the validated system model will deal with the system that corresponds with this signal-to-noise ratio. In Figure 9 the range profiles are plotted in the vertical direction while the horizontal axis indicates the time. The relation of time versus target distance or target aspect angle was shown in Figure 8 and is also indicated in the figure.

To identify the targets, the simulated data according to the scenario have to be compared with data in a database. Therefore, data for the databases have also been simulated using the model. The database consists of laser range profiles of the three aircraft for each 5 degrees of azimuth angle. Roll and pitch of the aircraft are not taken into account for the database.

\section{IDENTIFICATION RESULTS}

For identification, we use correlation of laser range profiles to compare sensor data with data in the database. Since the aspect angle of the aircraft is known from the track data, the simulated "measured" laser range profile is correlated with the closest range profile (in aspect angle) in the database. Before correlation the "measured" laser range profile (LRP) is scaled to the database LRP:

$$
L_{M}^{\prime}=S_{L} L_{M} \text { with } S_{L}=\frac{\sum L_{D B}}{\sum L_{M}},
$$

where $L_{M}$ is the "measured" LRP, $L_{D B}$ is the database LRP, and $S_{L}$ is the scaling factor. The maximum correlation is an indication whether the target corresponds to the target in the database:

$$
C_{M, D B}=\max \left[\sum L_{M}^{\prime}(\Delta x) L_{D B}\right] .
$$

This indication whether the target corresponds to the target in the database is expressed as a likelihood based on the correlation and the noise in the data:

$$
P=\exp \left[-F_{T} \frac{\left|\sum L_{M}^{\prime 2}+\sum L_{D B}^{2}-2 C_{M, D B}-N_{M} \sigma^{2} S_{L}^{2}\right|}{\sqrt{2 N_{M}} \sigma^{2} S_{L}^{2}}\right],
$$


where $F_{T}$ is a threshold factor, $N_{M}$ is the number of entries in the database LRP, and $\sigma$ is the noise in the "measured" LRP.

Figure 10 shows these likelihoods as images with the likelihoods plotted on a colour scale. The "measured" LRP's for the range of aspect angles between 0 and 360 degrees are based on the F15 database with added noise. As expected, the figure on the left for the F15 shows that the "measured" and database LRP correspond very well for identical azimuth angle. Note that the negative azimuth angle (or $360 \mathrm{deg}$ minus azimuth) also agrees quite well. This is due to the almost symmetrical shape of the F15. Likelihoods for the F16 and F117 are considerably smaller, since their LRP's do not correlate very well with the "measured" LRP's from the F15. Note that the colour scales differ, so that the maximum likelihoods of the F16 and F117 are 0.6 and 0.7, respectively; while the F15 has a maximum likelihood of 1.0. However, if we increase the noise, the likelihoods of these aircraft increase considerably as we shall show next.
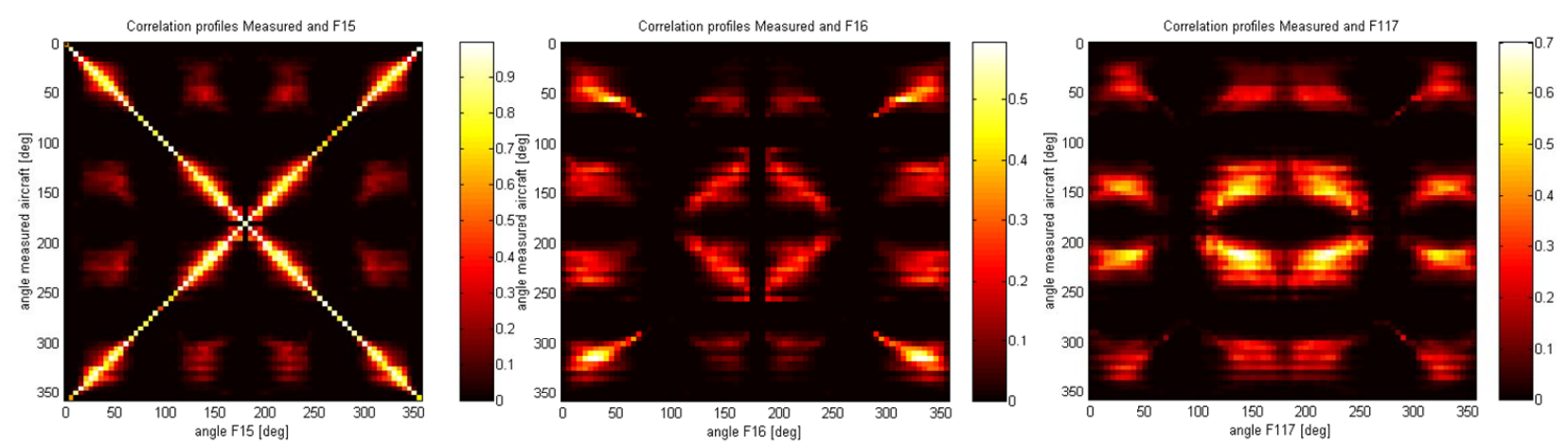

Figure 10: Likelihoods based on correlation of a "measured" LRP at various azimuth angles with database LRP's of F15 (left), F16 (middle), and F117 (right). Colour scales differ with maxima at 1.0, 0.6, and 0.7 for F15, F16, and F117, respectively.

Figure 11 shows the correlation and likelihood results for an approaching F16. At the start of the scenario, the F16 is at range of $120 \mathrm{~km}$ and the signal-to-noise ratio is low. All three aircraft have low correlation and high likelihood. Due to the large noise in the LRP's it is not possible to identify the approaching aircraft as an F16. After 250 seconds, the F16 is at a range of $30 \mathrm{~km}$ (see Figure 8) and the likelihood of the F16 exceeds those of the other two aircraft. Near the end of the scenario, the identification of the F16 fails for a moment. This is due to the side view of the aircraft. In side view the LRP's of the three aircraft are short and more or less similar.
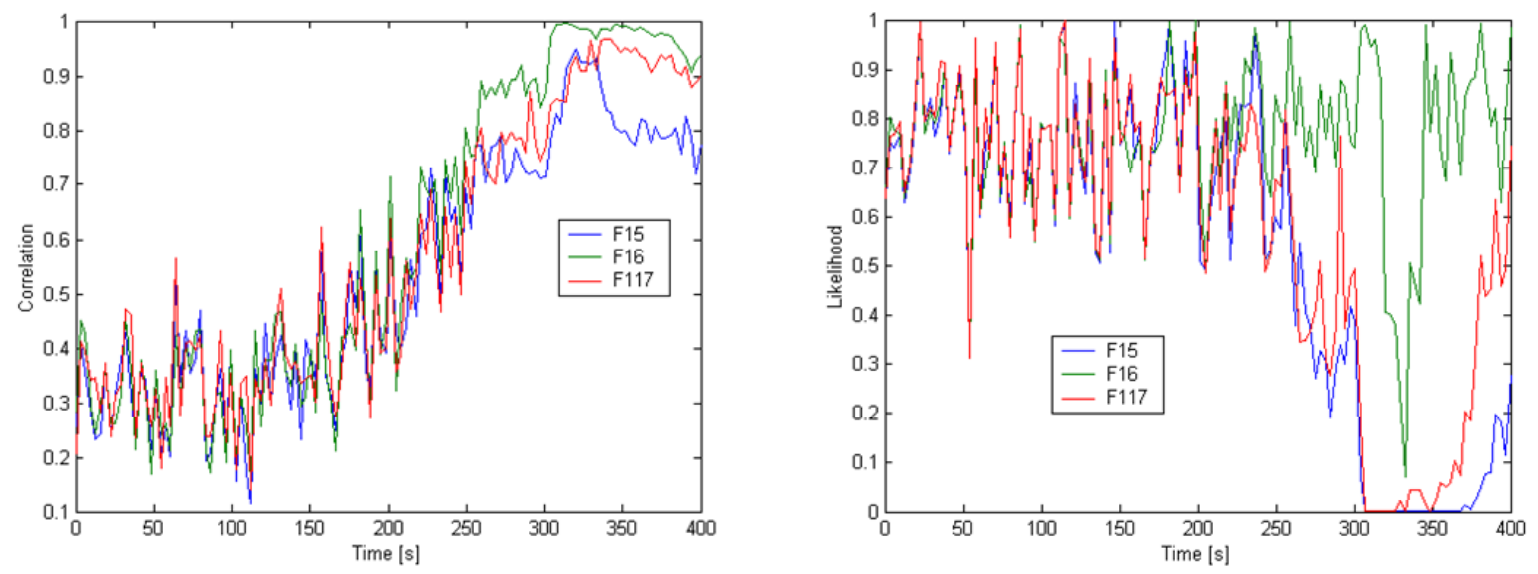

Figure 11: Correlation (left) and likelihood (right) for the three aircraft as a function of time in the scenario given an approaching F16.

The three correlations (for the three aircraft) give three likelihoods when taking the noise into account. A moderate correlation with large noise gives a high likelihood but a low one for little noise. These likelihoods are used to obtain 
probabilities using Bayes theorem. In the Bayes identification, we want to obtain the a-posteriori probability $P\left(A \mid L_{M}\right)$ that a class $A$ is present given a measured laser range profile $L_{M}$. The a-posteriori probability is given by

$$
P\left(A \mid L_{M}\right)=\frac{P\left(L_{M} \mid A\right) P(A)}{P\left(L_{M}\right)},
$$

where $P\left(L_{M} \mid A\right)$ is the likelihood given by the correlation, $P(A)$ is the a-priori probability that class $A$ occurs, and $P\left(L_{M}\right)$ is a normalization factor. This normalization factor determines that the sum over all classes of these a-posteriori probabilities is one. The a-priori probabilities can be based on intelligence ruling out certain aircraft.

A target class "other" has to be used for the case that an LRP is measured of an aircraft that is not in the database. Note that an aircraft type can also be in a certain configuration (weapons, fuel tank) that is not in the database. In both cases the aircraft should be classified as unknown and not as the wrong aircraft type. The problem is that $P\left(L_{M} \mid\right.$ other $)$ can not be determined from correlation of the LRP. In order to obtain the class "other" when all aircraft in the database give a low likelihood, we use a constant small value $\varepsilon$ for $P\left(L_{M} \mid\right.$ other $)$. To limit the number of free parameters, the a-priori probabilities will be set the same for all classes, effectively removing them. Now the normalization factor is given by

$$
P\left(L_{M}\right)=\sum_{\substack{\text { classes in } \\ \text { database }}} P\left(L_{M} \mid A\right)+\varepsilon,
$$

Figure 12 shows the result of the Bayesian identification. The figure is similar to the likelihoods of Figure 11, which is to be expected since all a-priori information is discarded. Again the F16 is identified at shorter ranges with a maximum probability of $95 \%$. The remaining $5 \%$ is claimed by the "other" class which is the consequence of the small value $\varepsilon$ of $5 \%$ for the likelihood of the "other" class $P\left(L_{M} \mid\right.$ other $)$.

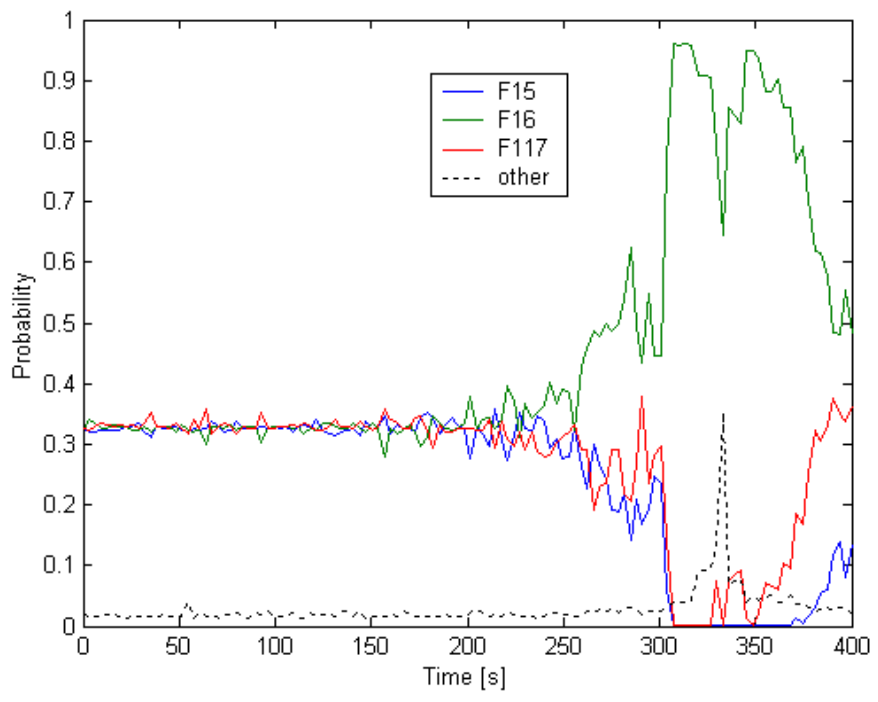

Figure 12: Bayes probabilities for an approaching F16 in the scenario.

The Bayes probability for the F16 reaches its maximum after 300 seconds from the start of the scenario. This is caused by the short range, which gives a good signal-to-noise ratio (SNR). According to the scenario, after around 320 seconds the distance between the two aircraft is at its minimum, about $5.6 \mathrm{~km}$. Some time later, after 350 seconds, the probability decreases due to the larger separation of the aircraft resulting in a deteriorating SNR. There is a striking local minimum in the probability at $330 \mathrm{~s}$ in the scenario. This is due to the aspect angle of the observed aircraft which is close to 90 degrees at that time. It is harder to distinguish aircraft at 90 degrees aspect angle since the range profile is very short and dominated by the peak from the side of the plane. 
For the identification of the aircraft, a Bayesian approach was followed without using a-priori information. However, it could be used in the described method to improve the performance and to combine with information from other sensors or sources. For instance, a-priori information can be derived from expectation based on historical records. In the presented method there was no learning or averaging of laser range profiles. Learning could be achieved by adjusting the a-priori information. When on basis of a-priori information an identification result is obtained, this information can be used to adjust the a-priori probabilities for a following identification.

We will now further investigate the relation between identification and aspect angle. As was illustrated in the scenario analysis, the identification range depends both on range and target aspect angle. It is possible to do the same analysis as previously with the scenario but now with a "measured" laser range profile from a rotating target. This analysis will yield a (Bayes) identification probability for the various aspect angles. Since we want to obtain the SNR for which the identification probability is at a certain threshold level, the above analysis is repeated for many different noise levels. With this data the noise level for each aspect angle is obtained that gives a fixed identification probability. Here we use an identification probability of $90 \%$. Since the SNR depends on the reciprocal squared range $\left(1 / \mathrm{R}^{2}\right)$, the required range for the $90 \%$ identification probability is proportional to the square root of the found noise level. Figure 13 shows the radial plots of the required range (in arbitrary units) against the aspect angle for an identification probability of $90 \%$.
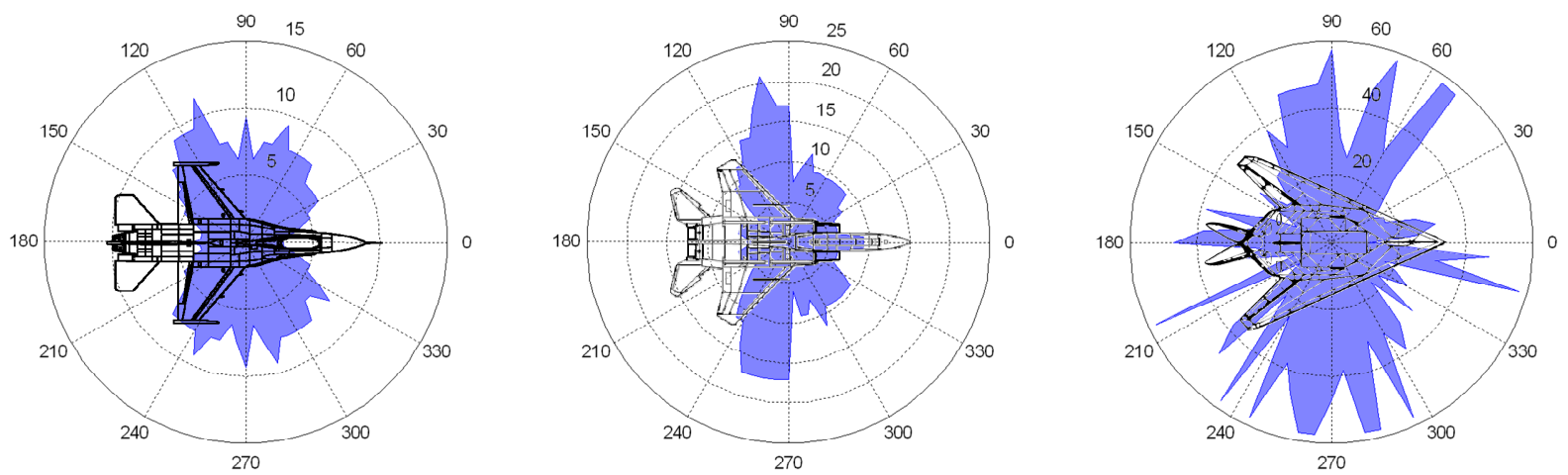

Figure 13: Radial plot corresponding to an identification probability of $90 \%$ for an F16, F15, and F117, left to right respectively.

It is clear form these plots that the required range is short for front view (aspect angle of zero degrees). This means that the aircraft has to be pretty close for correct identification. This is easily understood if we consider the aerodynamic shape of the aircraft that is responsible for a low laser return. We see a similar effect at 180 degrees aspect angle. Around 90 degrees there is a minimum in the radial plot of the three aircraft. This was discussed at the scenario analysis where it was seen as a drop in the probability at a certain time in the scenario. The reason is the difficulty to discriminate aircraft at 90 degrees aspect angle since the range profile is very short and dominated by the peak from the side of the plane.

It is also informative to compare the radial plots of the three aircraft. The F16 has a smaller maximum identification range than the F15 which has again a smaller range than the F117. Thus, the F117 can be identified at considerably longer ranges than the F16 on average. This is most likely due to the characteristic shape of the F117, which differs considerably from the more conventional aircraft: F16 and F15. Note that the longer identification range for the F117 does not mean that it can be detected at longer ranges. The range is about identification only. Detection is not an option since the very narrow laser beam can not search the azimuth and the elevation range in sufficiently short time.

\section{VALIDATION OF SYSTEM MODEL}

The system model calculates the receiver laser range profile. Starting point is the energy that is received from a small area of the target. The received energy is the integrated energy density over the aperture of the receiver given by 


$$
E_{R}=E_{T} \frac{\tau_{a t m}}{\pi(\varphi R)^{2}} \cdot \rho A_{T} \cos \theta \cdot \frac{A_{R}}{\pi R^{2}} \tau_{a t m} \tau_{s y s}
$$

where

$E_{T}$ is the (transmitted) laser pulse energy,

$\tau_{\text {atm }}$ is the atmospheric transmission (one-way),

$\varphi$ is the laser beam divergence (half angle),

$R$ is the range from target to receiver,

$\rho$ is reflectivity of the target,

$A_{T}$ is the target area,

$\theta$ is the incidence angle of the laser beam on target,

$A_{R}$ is the receiver area, and

$\tau_{\text {sys }}$ is the system transmission.

By using this equation for every point of the 3D model, we get a distribution (histogram) of energies in consecutive time intervals. These represent a laser range profile in received laser power versus time. Note that each 3D point in the simulation will have a different range. In the simulation we will use the system parameters of Table 2 .

Table 2 Simulation parameters for the laser range profile of a ship at $8.5 \mathrm{~km}$.

\begin{tabular}{|l|l|}
\hline Parameter & Value \\
\hline Laser pulse energy & $30 \mathrm{~mJ}$ \\
\hline Atmospheric transmission & 0.33 \\
\hline Laser divergence & $1 \mathrm{mrad}$ \\
\hline Range to target & $8.5 \mathrm{~km}$ \\
\hline Reflectivity target & 0.5 \\
\hline Receiver area & $28 \mathrm{~cm}^{2}$ \\
\hline System transmission & 0.5 \\
\hline Noise equivalent power & $14 \mathrm{nW}$ \\
\hline
\end{tabular}

The system model was validated with experimental results from the experiments described in previous sections. For the validation, we used the laser range profile of a frigate-sized ship at $8.5 \mathrm{~km}$. Figure 14 shows that the experimental and the simulated profile correspond quite well.

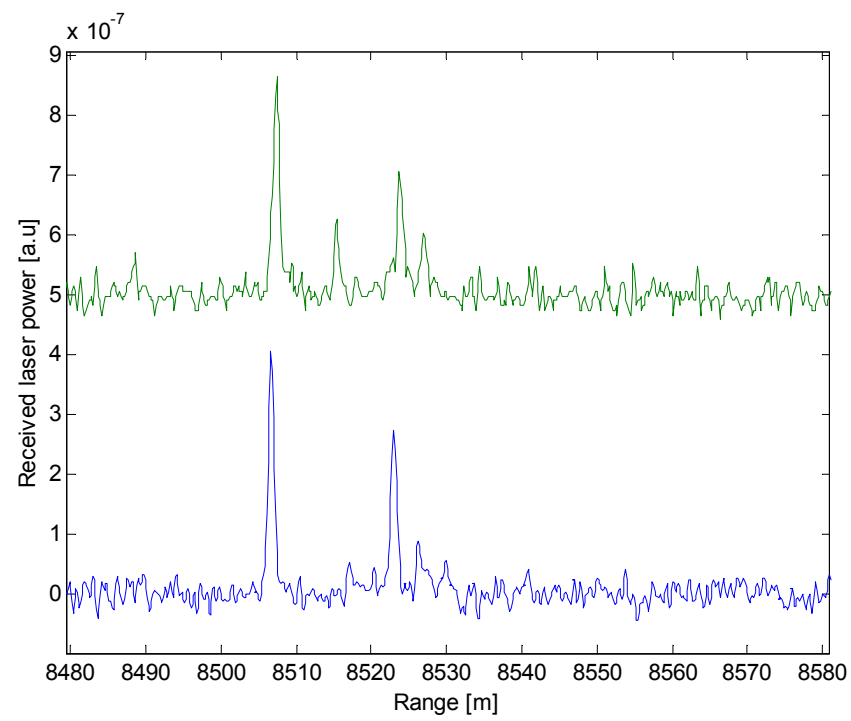

Figure 14: Simulated (bottom) and experimental (top) laser range profile. 
We will now compare this system model with the simulation for air-target identification. In the scenario we have simulated the noise, which is shown in Figure 15 for the time of $300 \mathrm{~s}$ after the start of the scenario when the F16 is viewed straight at the front at a range of about $8 \mathrm{~km}$. The SNR is about 0.5 , i.e. the noise is about twice the profile (standard deviation noise versus mean of profile). This SNR corresponds with the system parameters of Table 2 except for the atmospheric transmission that is 1.0 in accordance with the excellent transmission at high altitude.

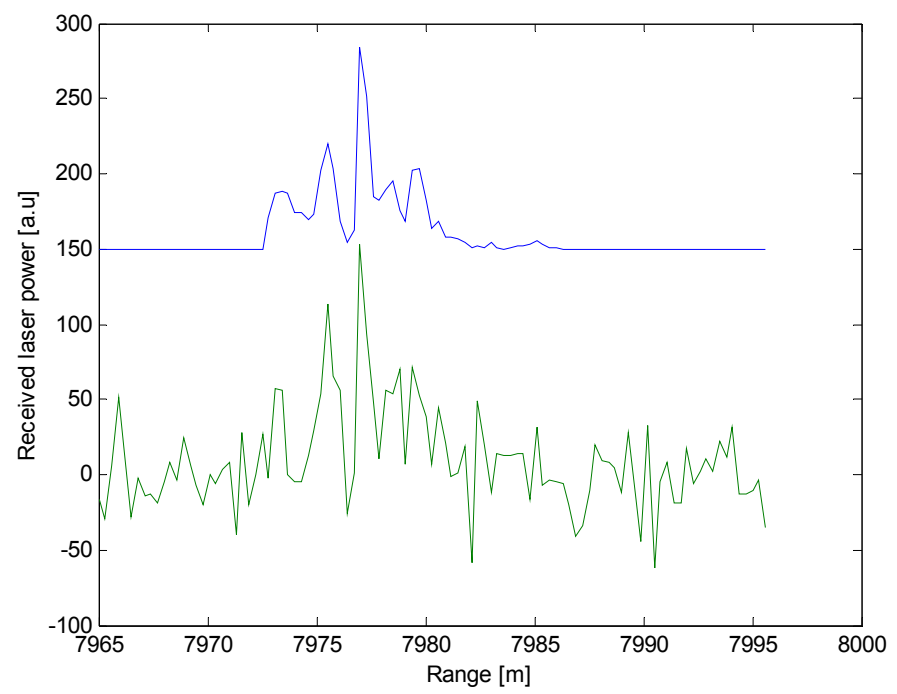

Figure 15: Original (top) and scenario (bottom) laser range profile of the F16 in the scenario after $300 \mathrm{~s}$ in a front view position.

The system of Table 2 is clearly not very useful for air-target identification. However, a much better system can be envisioned. For a start, the system parameters leave room for improvement. The NEP (noise equivalent power) of the detector can be improved up to a factor of five. In addition, the laser pulse energy and the receiver area can be increased. Perhaps it is also feasible to reduce the beam divergence for a higher power density on target. In the simulation the beam size had a diameter of about 16 meter at the range of $8 \mathrm{~km}$ compared to a wingspan of $10 \mathrm{~m}$. It should be noticed that we implicitly used a varying beam divergence in the scenario simulation, since we used an SNR proportional on $1 / R^{2}$. For a fixed divergence, this should be $1 / R^{4}$. If we consider the possible improvements listed above, the improved signal-to-noise ratio (SNR) could amount to a factor of 25; a factor of five for the SNR of the receiver times a factor of five for laser pulse energy and receiver area. This total increase in SNR of a factor 25 means that the maximum range is increased a factor five $\left(1 / \mathrm{R}^{2}\right)$. Since in the simulation good results were obtained at $8 \mathrm{~km}$, a maximum range of five times that range, namely $40 \mathrm{~km}$, is possible. Note that the beam divergence should be decreased with a similar amount, i.e. reduced to $0.2 \mathrm{mrad}$. This divergence is not a problem; however, the pointing has to be that accurate as well. This seems feasible though. In the simulation we also found that at a range of $30 \mathrm{~km}$ the F16 is preferred above the other two aircraft, with the improved system this would occur at a range of $150 \mathrm{~km}$. An increased SNR at this range, and thus a more reliable identification, could be obtained by averaging laser profiles from different laser pulses.

The system of Table 2 is an eye-safe system at 1.5 micron. However, much better ranges can be achieved by a laser at 1.06 micron. In this case the detector is more sensitive and higher pulse energies are obtainable. The system will not be eye-safe at the aperture, however, it could be eye-safe at the air target.

\section{SUMMARY AND CONCLUSIONS}

We have presented a concept for identification of sea-surface targets using a laser range profiler. This concept has been evaluated using real laser range profile data from a NATO trial in Norway. Results of this trial show that a laser range 
profiler can measure profiles of ships up to a range of $10 \mathrm{~km}$. It is shown that these profiles match the geometry of the ship very closely and are distinctive provided that the shapes of the ships are not the same.

An important factor is the database. For good identification results, a detailed database is needed for all ships that are of interest. In practice, it will be difficult to obtain such a database by measurements alone. An option is to use modeling results. It has been shown here that there is a good correspondence between experimental and simulated profiles if the $3 \mathrm{D}$ model is accurate.

We have presented a concept for air-to-air identification using a laser range profiler. This concept has been evaluated and demonstrated using modelled sensor data. It is shown that the Bayesian identification gives good identification results when the signal-to-noise ratio is not too low. The experimental results confirmed the simulation assumptions of good range resolution. In addition, the system model used in the simulation of air-target identification has been validated in the experiments of ship identification. The system model indicated that it is possible with existing state-ofthe-art technology to reach a maximum identification range of several tens of kilometers.

\section{ACKNOWLEDGEMENTS}

We thank LtCdr Eric in 't Veld and Lt Hans Daams for facilitating the laser range profiling measurements at the MCG/8 trial. We further thank Wim Pelt and Lt. Rob Klijn of the Netherlands Ministry of Defence for supporting this work in programs V404 and V510. Dr. Dolf Bos is thanked for support in program V504.

\section{REFERENCES}

1. Heiden, R. van der, [Aircraft Recognition with Radar Range Profiles], Thesis University of Amsterdam, (1998).

2. Berkowitz, R.S., [Modern Radar - Analysis, Evaluation, and System Design], John Wiley \& Sons, Inc., (1965).

3. Koenderink, J.J., Doorn, A.J. van, "Bidirectional Reflection Distribution Function of Thoroughly Pitted Surfaces", Int. J. Comp. Vision 31(2/3), 129-144 (1999).

4. Beckmann, P., Spizzichino, A., [The scattering of Electromagnetic Waves from Rough Surfaces], Pergamon Press, (1963).

5. Torrance, K., Sparrow, E., “Theory for off-specular reflection of roughened surfaces”, J. Opt. Soc. Am. 57, 11051114 (1967).

6. Kunz, G.J., et al., "Status and developments in EOSTAR, a model to predict IR sensor performance in the marine environment", Proc. SPIE Vol. 5572-12, pp. 1-12 (2004).

7. Kunz, G.J., et al., "Detection of small targets in a marine environment using laser radar", Proc. SPIE Vol. 5885 (2005).

8. Heuvel, J.C. van den, et al., "Identification of Littoral Targets with a Laser Range Profiler". Proc. SPIE Vol. 6550 (2007).

9. Broek, A.C. van den, et al., "A multi-sensor scenario for coastal surveillance". Proc. SPIE Europe Remote Sensing, 17-20 September 2007 in Florence, Italy (2007).

10. Sutherland, J., HNeT, AND corporation, www.andcorporation.com. 